\title{
Diagrams increase the recall of nondepicted text when understanding is also increased
}

\author{
Michael J. SERRA \\ Texas Tech University, Lubbock, Texas
}

\begin{abstract}
Multimedia presentations typically produce better memory and understanding than do single-medium presentations. Little research, however, has considered the effect of multimedia on memory for nonmultimedia information within a large multimedia presentation (e.g., nondepicted text in a large text with diagrams). To this end, the present two experiments compared memory for target text information that was either depicted in diagrams or not. Participants $(n=180)$ studied either a text-only version of a text about lightning or a textwith-diagrams version in which half the target information was depicted in diagrams. Memory was tested with both free recall and cued recall questions. Overall, diagrams did not affect memory for the entire text; diagrams increased memory only for the information they depicted. Diagrams exerted a generalized effect on free recall only when diagrams increased the overall understanding of the text (i.e., when the participants studied the materials twice before the test).
\end{abstract}

The term multimedia is commonly used to describe a variety of interactive digital media, such as video games, virtual reality, hypermedia (e.g., the World-Wide Web), interactive art installations, and the CD-ROMs that accompany many textbooks (Packer \& Jordan, 2001). As defined by multimedia-learning researchers, multimedia refers to the presentation of information visually and verbally at the same time, using words and pictures (e.g., a text with diagrams; Mayer, 2009). Such multimedia presentations typically produce better memory and understanding for the topic presented than either of the single-medium components would alone (e.g., text alone or diagrams alone; Mayer, 2009).

One model that has been developed to account for the effects of multimedia on memory and understanding is Mayer's (2009) cognitive theory of multimedia learning (CTML). The CTML assumes that visual and verbal information are processed in two separate channels, each with its own limited processing capacity. The theory also assumes that learning is an active process in which the learner tries to understand and remember the information presented. The CTML proposes that when information is presented both visually and verbally at the same time (as in a multimedia presentation), more connections can be formed between the information than can be formed when information is presented in a single medium. These connections are formed as the reader selects relevant visual and verbal information from the presentation, organizes it into separate visual and verbal models of the scenario being conveyed by the presentation, and finally integrates the two representations with each other and with prior knowledge (Mayer, 2009). The greater number of connections made in a multimedia presentation, relative to a single-medium presentation, is presumably responsible for the increase in both memory and understanding.

The CTML is compatible with Kintsch's (1988) construction-integration model of text comprehension, which posits three levels of text comprehension. The lexical level involves encoding the surface features of a text (i.e., words and syntax). The textbase level involves encoding the surface features into propositions and forming links between propositions. The situation model involves linking propositions with prior knowledge in the reader's long-term memory. The creation of a text representation proceeds in cycles at all three levels, with component processes operating on text segments one at a time. On the basis of this model, at least three factors can affect memory for target text (Rawson \& Kintsch, 2004): (1) whether the target information has been encoded, (2) whether connections between the target information and other pieces of information have been encoded, and (3) whether retrieval cues are available for the target information. Diagrams might affect memory in any, or all, of these ways. For example, including diagrams with text might increase the likelihood that depicted information is encoded, make participants more likely to understand how depicted information relates to other information in a passage, and provide better internal recall cues for depicted information than for nondepicted information.

To evaluate predictions from the CTML, many studies on text with diagrams report retention of the text information that is depicted in the diagrams of a multimedia condition. Typically, retention of this information is compared with retention of the same information by a text-only group. Consider, however, that in actual learning situations, a goal is often to learn more information than is just 
depicted in diagrams. Nevertheless, few studies report retention of information that was not presented in diagrams to either group. For this reason - as well as for the continued refinement of theories of multimedia learning - it is critical to consider how multimedia affects memory for related information that is not actually depicted in both media. Intuitively, one might predict that only memory for the information depicted in the diagrams will benefit, because only that information will be strengthened by the visual depiction. By contrast, one intriguing prediction from CTML is that diagrams will increase retention for the entire text, not just for the information present in the diagrams. Such a prediction is supported by theories of text comprehension, such as construction-integration (Kintsch, 1988; Rawson \& Kintsch, 2004), that focus on the holistic processing of the entire text.

One study that actually reported retention of nondepicted information in a multimedia-learning situation was Waddill and McDaniel (1992). The participants in that study read either a text-only version of a text about avalanches (control group) or one of two versions that included diagrams; they also rated their understanding for the text. The main dependent variable of interest in the study was performance on a surprise free recall test. Diagrams increased the recall of depicted information, as compared with the recall of the same information by participants in the text-only group, but did not affect recall of the nondepicted information. That study, however, was not conducted for such purposes, and elements of its design make this comparison less than ideal. Specifically, the participants studied the materials under incidental learning conditions, and the information that was not depicted in any of the diagrams was detail information that did not factor strongly into the overall explanation of how avalanches occur. This information was rated as being less important than the depicted information, which is why it was chosen not to be depicted with diagrams in the multimedia conditions. It is therefore possible that the results of Waddill and McDaniel would not generalize to intentional learning situations when memory for important, nondepicted information is tested, because both the CTML and construction-integration assume that learners are studying the materials to learn them and that all of the target information is relevant to comprehending the text as a whole.

To this end, the main goal of the present study was to examine further how adding diagrams to text affects the retention of nondepicted information. The participants in the present experiments studied either a text-only or a multimedia (text with diagrams) presentation on how lightning storms develop. Retention of both the depicted and nondepicted information was tested with free recall and cued recall (i.e., short-answer) questions. Given the theories above, one might expect an overall generalized effect on all retention measures, because all of the information presented (whether in diagrams or not) is causally related and likely to be processed holistically. Alternatively, a multimedia effect might occur for the free recall but not the cued recall of nondepicted information. Whereas free recall would be expected to take advantage of the integrated processing of the entire text, cued recall would not. At test, cues such as short-answer questions are likely to cue only the specific information being asked about and not other parts of the text or diagrams (Rawson $\&$ Kintsch, 2004). Thus, if the short-answer questions do not also cue information that was depicted in the diagram, the cued recall of nondepicted information may not benefit from multimedia encoding.

\section{EXPERIMENT 1}

\section{Method}

\section{Participants and Design}

One hundred undergraduates from Kent State University participated in this study for course credit. Experiment 1 utilized a 2 (format: text only vs. multimedia) $\times 2$ (test type: free recall vs. cued recall) $\times 2$ (depiction: depicted vs. nondepicted) mixed design. Presentation format was manipulated between participants. The participants were randomly assigned to either study group. Test and depiction types were within-participants independent variables.

\section{Materials}

Study materials. The study materials were a 500 -word science text with diagrams about how lightning storms develop, adapted from Mayer (2009). The text had six text paragraphs that were used in both the multimedia and text-only groups. In the multimedia group, a corresponding full-color diagram accompanied each paragraph. Figure 1 shows an example paragraph with its corresponding diagram. The diagrams did not add any additional information to the paragraphs. Rather, each diagram illustrated one of the two pieces of target information contained in the corresponding text paragraph. To make discussion of these sets of information easier, the information that never appeared in any diagrams for either group will be referred to as nondepicted and the information that appeared in the diagrams in the multimedia group will be referred to as depicted. The retention of the depicted and nondepicted information was considered separately in both groups (and labeled as depicted) for comparison purposes even though, in the text-only group, both sets of information were presented without diagrams.

Retention measures. Memory for all 12 pieces of information was tested in both groups with free recall and cued recall questions. The participants' retention of the study materials was tested with one free recall question ("In the box below, please type an explanation of how lightning works"). To answer this question, the participants were to explain, giving as much information as they could, how lightning storms develop. Responses were scored on the basis of how many of the 12 pieces of information they recalled. The participants' cued recall of the same 12 pieces of information was tested with 12 cued recall (short-answer) questions. The top of Table 1 contains the two target pieces of information found in Figure 1; successful free recall of this information would be counted into the total free recall scores.

Table 1

Example of Retention Measures for Figure 1

\section{Target Information for Free Recall}

Dart leaders carry negatively-charged particles from the cloud down the path of the previous stroke [depicted]

Multiple dart leaders coming down from the cloud make it look like the lightning is flickering [nondepicted]

Cued Recall Questions and Answers

Q: What do dart leaders carry down from the cloud?

A: Dart leaders carry negatively-charged particles [depicted].

Q: Why does lightning appear to flicker?

A: Multiple dart leaders come down from the cloud [nondepicted].

Note-Labels indicate whether the target information was depicted for the multimedia groups or not. 


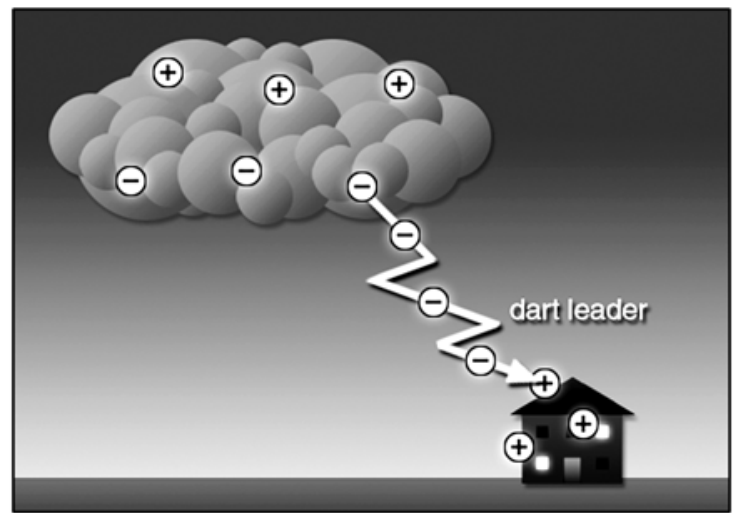

A flash of lightning may end after one return stroke. In most cases, however, dart leaders (which are similar to stepped leaders) carry more negative charges from the cloud down the main path of the previous stroke. Each dart leader is followed by a return stroke. This process commonly occurs 3 or 4 times in one flash, but can occur more than 20 times. People can sometimes see the individual strokes of a flash. At such times the lightning appears to flicker.

Figure 1. Example of a diagram-paragraph pair used in Experiments 1 and 2. Each participant studied a six-screen presentation on how lightning storms develop that contained either six text paragraphs (text-only groups) or the same six text paragraphs with accompanying full-color diagrams (multimedia groups).

The bottom of Table 1 contains two cued recall questions designed to test cued recall of the same information.

Transfer questions. The participants' understanding of the study materials was tested with three long-answer transfer questions (see Mayer, 2009). Answering these questions required the participants to understand the study materials, since the answers were not explicitly stated in the text (e.g., Q: "What could you do to decrease the intensity of lightning?" A: "Reduce the number of negative ions in storm clouds").

\section{Procedure}

The procedure in Experiment 1 was the same for the two groups, except that a diagram accompanied each paragraph in the multimedia group. Instances of both types of materials will henceforth be referred to as screens. In the text-only group, each screen contained a single text paragraph; in the multimedia group, each screen contained a single text paragraph and a single corresponding diagram. Up to 4 participants at a time completed the study on separate PCs in the same room. These computers displayed the materials, instructions, and questions to the participants and recorded all responses and latencies. The computers presented the six screens one at a time to the participants in serial order, but the participants could move back and forth between the screens by clicking "forward" and "back" icons on the screens. After the sixth screen had been viewed, an additional icon appeared on the screen that allowed the participants to proceed to the test. The participants were instructed to study the material until they felt that they understood it enough to answer questions on it later. No restrictions were placed on the study phase, but the participants could not return to the study materials once they opted for the test. The participants then completed the tests on the computer. They first answered the free recall question, then the 12 cued recall retention questions in a random order, and finally the 3 transfer questions in a fixed order. The participants had an unlimited amount of time to answer each question but could not go back to a question once it had been answered or left blank.

\section{Results and Discussion}

\section{Study Time and Transfer Performance}

The measure of study time was the mean total time the participants spent studying the six screens. Mean study times are presented in Table 2. Study time was not different for the two groups $\left[F(1,98)=0.6, M S_{\mathrm{e}}=44,456.3\right.$, $p>.05]$. Similarly, the mean number of times the participants studied each screen was not different for the two groups [multimedia, $M=1.7$ times/screen, $S D=0.8$; text only, $M=1.7$ times/screen, $S D=0.9 ; F(1,98)=0.0$, $\left.M S_{\mathrm{e}}=0.7, p>.05\right]$.

Performance on the transfer questions was not different for the two groups [multimedia, $M=1.3, S D=0.87$; text only, $M=1.2, S D=1.0 ; F(1,98)=0.18, M S_{\mathrm{e}}=0.88$, $p>.05]$. These data suggest that the diagrams did not enhance the participants' understanding of the text.

\section{Retention Measures}

Performance on the retention questions (free recall and cued recall) was split on the basis of whether the target information was presented either just in the text or in both the text and diagrams in the multimedia group. Free recall was scored on the basis of how many of the 12 pieces of information (6 depicted and 6 nondepicted) from the text were recalled. Responses to the cued recall questions ( 6 depicted and 6 nondepicted questions) were scored based on how many were answered correctly. Percentages correct for the two subsets of questions are presented in Table 3.

Table 2

Mean Study Times (in Seconds) in Experiments 1 and 2

\begin{tabular}{|c|c|c|c|c|c|}
\hline \multirow{2}{*}{$\begin{array}{c}\text { Study } \\
\text { Trial }\end{array}$} & \multicolumn{2}{|c|}{$\begin{array}{l}\text { Text-Only } \\
\text { Group }\end{array}$} & \multicolumn{2}{|c|}{$\begin{array}{l}\text { Multimedia } \\
\text { Group }\end{array}$} & \multirow[b]{2}{*}{ ES } \\
\hline & $M$ & $S D$ & $M$ & $S D$ & \\
\hline \multicolumn{6}{|c|}{ Experiment 1} \\
\hline Trial 1 & 398.3 & 248.4 & 366.2 & 165.0 & 0.15 \\
\hline \multicolumn{6}{|c|}{ Experiment 2} \\
\hline Trial 1 & 288.6 & 96.6 & 344.1 & 122.6 & 0.50 \\
\hline Trial 2 & 211.8 & 73.0 & 205.3 & 69.0 & 0.09 \\
\hline
\end{tabular}

Note-Study times represent the total time spent studying the six screens. "ES" refers to the effect size (Cohen's $d$ ) of the difference between the two means.

Table 3

Descriptive Statistics in Experiment 1: Percentages Recalled

\begin{tabular}{|c|c|c|c|c|c|}
\hline \multirow{2}{*}{$\begin{array}{c}\text { Information } \\
\text { Type }\end{array}$} & \multicolumn{2}{|c|}{$\begin{array}{l}\text { Text-Only } \\
\text { Group }\end{array}$} & \multicolumn{2}{|c|}{$\begin{array}{l}\text { Multimedia } \\
\text { Group }\end{array}$} & \multirow[b]{2}{*}{ ES } \\
\hline & $M$ & $S D$ & $M$ & $S D$ & \\
\hline \multicolumn{6}{|c|}{ Free Recall } \\
\hline Depicted & 33.4 & 30.0 & 49.6 & 39.0 & 0.47 \\
\hline Nondepicted & 28.7 & 26.1 & 35.3 & 30.2 & 0.23 \\
\hline \multicolumn{6}{|c|}{ Cued Recall } \\
\hline Depicted & 41.7 & 22.8 & 63.0 & 29.4 & 0.81 \\
\hline Nondepicted & 37.7 & 24.9 & 39.0 & 27.7 & 0.05 \\
\hline
\end{tabular}

Note- "Nondepicted" refers to information not depicted in the diagrams of the multimedia group. "Depicted" refers to information depicted in the diagrams of the multimedia group. The text-only group studied all information without any diagrams. "ES" refers to the effect size (Cohen's $d$ ) of the difference between the two means. 
Performance on the two retention measures was analyzed with a 2 (format: text only vs. multimedia) $\times 2$ (test type: free recall vs. cued recall) $\times 2$ (depiction: depicted vs. nondepicted) mixed ANOVA. The main effect of format was significant $\left[F(1,98)=5.5, M S_{\mathrm{e}}=2370.2\right.$, $\left.p<.05, \eta_{\mathrm{p}}^{2}=.1\right]$, as were the main effects of test type $\left[F(1,98)=13.8, M S_{\mathrm{e}}=535.9, p<.001, \eta_{\mathrm{p}}^{2}=.1\right]$ and depiction $\left[F(1,98)=44.9, M S_{\mathrm{e}}=307.8, p<.001, \eta_{\mathrm{p}}^{2}=.3\right]$. Depiction interacted with format $\left[F(1,98)=17.7, M S_{\mathrm{e}}=\right.$ $\left.307.8, p<.001, \eta_{\mathrm{p}}^{2}=.2\right]$.

The omnibus ANOVA was followed by separate comparisons so as to flesh out the effects of diagrams on memory for depicted and nondepicted information. As might be expected, recall of depicted information was higher for the multimedia group than for the text-only group $[F(1,98)=$ $\left.5.5, M S_{\mathrm{e}}=1,212.2, p<.05, \eta_{\mathrm{p}}^{2}=.1\right]$. The two groups, however, recalled equal amounts of the nondepicted information $\left[F(1,98)=1.4, M S_{\mathrm{e}}=795.0, p>.05\right]$. Similarly, cued recall performance for nondepicted information was equal for the two groups $\left[F(1,98)=0.1, M S_{\mathrm{e}}=692.7, p>\right.$ $.05]$, and cued recall performance for depicted information was higher for the multimedia group than for the text-only group $\left[F(1,98)=16.3, M S_{\mathrm{e}}=691.6, p<.001, \eta_{\mathrm{p}}^{2}=.1\right]$. The results of Experiment 1 demonstrate that - at least when understanding is equal across the two groups - the effect of diagrams on retention is limited to the information depicted in the diagrams for both free and cued recall.

\section{EXPERIMENT 2}

Given the assumptions of the CTML and constructionintegration, it seems likely that a generalized effect of diagrams on memory would occur only if a situation-model (i.e., well-integrated) level of understanding were achieved. Thus, the failure to find generalized effects in Experiment 1 may have arisen because multimedia effects did not influence transfer performance, which is considered a measure of understanding (Mayer, 2009). To evaluate this possibility, the procedure was changed to increase the likelihood that a multimedia effect would occur on transfer performance. In particular, the participants studied the materials twice before moving on to the test. Studying the materials twice should increase all the participants' understanding of the materials (Mayer, 1983) and better allow them to form a situation-model-level understanding of the text as a whole (Millis, Simon, \& tenBroek, 1998; Rawson $\&$ Kintsch, 2004). Thus, this procedure should provide a better test of whether or not diagrams exert selective effects on retention (free recall and cued recall).

\section{Method}

\section{Participants, Design, and Materials}

Eighty undergraduates from Kent State University participated in this study for course credit; none had participated in Experiment 1. The design, study materials, and test questions used in Experiment 2 were the same as those used in Experiment 1.

\section{Procedure}

The procedure was the same as that in Experiment 1, except that, in Experiment 2, the participants in both groups studied the materials twice in succession before being tested over the materials.
The participants were given 5 min between the two study periods to complete an unrelated puzzle to increase the likelihood that they would benefit from studying the materials again. The participants in Experiment 2 could study each screen of the presentation for as long as they wanted, but once a screen was studied, they could not go back to study it again. The test procedure was the same as that in Experiment 1.

\section{Results and Discussion}

\section{Study Time and Transfer Performance}

Mean study times are presented in Table 2. Study time was considered separately for the two study opportunities and was entered into a 2 (presentation format) $\times 2$ (trial) mixed ANOVA. Presentation format did not affect overall study time $\left[F(1,78)=2.3, M S_{\mathrm{e}}=10,564.7, p>.05\right]$. The participants spent less time studying on the second trial than on the first trial $\left[F(1,78)=69.7, M S_{\mathrm{e}}=6,667.5, p<\right.$ $\left..001, \eta_{\mathrm{p}}^{2}=.5\right]$. The interaction was significant $[F(1,78)=$ $\left.5.8, M S_{\mathrm{e}}=6,667.5, p<.05, \eta_{\mathrm{p}}^{2}=.1\right]$. Follow-up ANOVAs showed that, on the first study trial, study time was greater for the multimedia group than for the text-only group $\left[F(1,78)=5.1, M S_{\mathrm{e}}=12,189.7, p<.05, \eta_{\mathrm{p}}^{2}=.1\right]$; on the second study trial, study time was not different for the two groups $\left[F(1,78)=0.2, M S_{\mathrm{e}}=5,042.5, p>.05\right]$.

Transfer performance was greater for the multimedia group $(M=2.0, S D=.96)$ than for the text-only group $(M=1.3, S D=.88)\left[F(1,78)=9.9, M S_{\mathrm{e}}=.85, p<.005\right.$, $\left.\eta_{\mathrm{p}}^{2}=.11\right]$. These data suggest that the diagrams enhanced the participants' understanding of the text.

\section{Retention Measures}

Percentages correct for the two subsets of questions for the two retention measures are presented in Table 4. Performance on the two retention measures was analyzed with a 2 (format: text-only vs. multimedia) $\times$ 2 (test type: free recall vs. cued recall) $\times 2$ (depiction: depicted vs. nondepicted) mixed ANOVA. The main effect of format was significant $\left[F(1,78)=18.1, M S_{\mathrm{e}}=\right.$ $\left.1481.8, p<.001, \eta_{\mathrm{p}}^{2}=.2\right]$, as were the main effects of test type $\left[F(1,78)=7.8, M S_{\mathrm{e}}=608.5, p<.01, \eta_{\mathrm{p}}^{2}=.1\right]$ and depiction $\left[F(1,78)=47.7, M S_{\mathrm{e}}=366.7, p<.001\right.$, $\left.\eta_{\mathrm{p}}^{2}=.4\right]$. Information type interacted with presentation format $\left[F(1,78)=19.0, M S_{\mathrm{e}}=366.7, p<.001, \eta_{\mathrm{p}}^{2}=.2\right]$,

Table 4

\begin{tabular}{|c|c|c|c|c|c|}
\hline \multirow{2}{*}{$\begin{array}{c}\text { Information } \\
\text { Type }\end{array}$} & \multicolumn{2}{|c|}{$\begin{array}{l}\text { Text-Only } \\
\text { Group }\end{array}$} & \multicolumn{2}{|c|}{$\begin{array}{l}\text { Multimedia } \\
\text { Group }\end{array}$} & \multirow[b]{2}{*}{ ES } \\
\hline & $M$ & $S D$ & $M$ & $S D$ & \\
\hline \multicolumn{6}{|c|}{ Free Recall } \\
\hline Depicted & 33.9 & 27.8 & 59.9 & 31.9 & 0.87 \\
\hline Nondepicted & 32.1 & 22.5 & 45.5 & 28.2 & 0.53 \\
\hline \multicolumn{6}{|c|}{ Cued Recall } \\
\hline Depicted & 46.7 & 27.8 & 75.8 & 18.1 & 1.20 \\
\hline Nondepicted & 37.5 & 24.1 & 42.1 & 22.6 & 0.20 \\
\hline
\end{tabular}

Note- "Nondepicted" refers to information not depicted in the diagrams of the multimedia group. "Depicted" refers to information depicted in the diagrams of the multimedia group. The text-only group studied all information without any diagrams. "ES" refers to the effect size (Cohen's $d$ ) of the difference between the two means. 
and test and depiction interacted $\left[F(1,78)=19.0, M S_{\mathrm{e}}=\right.$ $\left.188.1, p<.001, \eta_{\mathrm{p}}^{2}=.2\right]$.

The omnibus ANOVA was followed by separate comparisons to further examine the effects of diagrams on memory for depicted and nondepicted information. Unlike in Experiment 1, recall of nondepicted information was higher for the multimedia group than for the text-only $\operatorname{group}\left[F(1,78)=5.5, M S_{\mathrm{e}}=651.5, p<.05, \eta_{\mathrm{p}}^{2}=.1\right]$. Recall of depicted information was also higher for the multimedia group than for the text-only group $[F(1,78)=$ $\left.15.2, M S_{\mathrm{e}}=897.6, p<.001, \eta_{\mathrm{p}}^{2}=.2\right]$. When diagrams improve understanding, therefore, they can also exert a general effect on free recall. As in Experiment 1, however, cued recall of nondepicted information was not different for the two groups in Experiment $2\left[F(1,78)=0.8, M S_{\mathrm{e}}=\right.$ $546.6, p>.05]$. In contrast, cued recall of depicted information was higher for the multimedia group than for the text-only group $\left[F(1,78)=31.0, M S_{\mathrm{e}}=549.5, p<\right.$ $\left..001, \eta_{\mathrm{p}}^{2}=.3\right]$. Therefore, a general effect of diagrams on retention obtained with free recall but not cued recall when diagrams also increased the understanding of the multimedia group over the text-only group.

\section{GENERAL DISCUSSION}

The present study examined how diagrams affect memory for nondepicted text information. The participants studied either text alone or a text with diagrams on how lightning storms develop. Retention of both the depicted and nondepicted information was tested with free recall and cued recall questions. Multimedia effects on retention were consistently found for depicted information, which would be expected from previous studies (see Mayer, 2009, for a review). By contrast, multimedia effects for nondepicted information (1) were never significant for cued recall questions and (2) arose in free recall for nondepicted information only when diagrams also boosted transfer performance (Rawson \& Kintsch, 2004).

Although these results might not be entirely predicted by current theories of multimedia learning, they are in line with some of the secondary results of Waddill and McDaniel (1992). More specifically, Waddill and McDaniel found that although diagrams did not increase the free recall of nondepicted (detail) information overall, relational diagrams in their study did increase the free recall of nondepicted information for their best readers (but not for their poorest readers). One possibility is that the multimedia format helped only their better readers to develop a situation model for the text. As with the results of the present study, this suggests that multimedia might increase the recall of nondepicted information only when the text has been understood well. But why might this be?

As was previously noted, the construction-integration model of text comprehension predicts that memory for the target text can be affected by factors such as whether connections between the pieces of target information have been encoded and whether retrieval cues are available for the target information (Kintsch, 1988; Rawson \& Kintsch, 2004). Although diagrams proved to be a consistent way to increase the likelihood of both free and cued recall for depicted information, diagrams increased free recall for nondepicted information only in Experiment 2, in which understanding (i.e., transfer performance) was also increased. This likely obtained because free recall takes advantage of the integrated processing of the entire text, whereas cued recall benefits less from such integration (cf. Rawson \& Kintsch, 2004). Further exploring these mechanisms and the link between understanding and retention within a multimedia presentation is likely to be a fruitful avenue for future research.

\section{AUTHOR NOTE}

The results from this study were presented at the 115th Annual Meeting of the American Psychological Association and at the 80th Annual Meeting of the Midwestern Psychological Association. Correspondence concerning this article should be addressed to M. J. Serra, Department of Psychology, Texas Tech University, MS 2051, Psychology Building, Lubbock, TX 79409-2051 (e-mail: michael.serra@ttu.edu).

\section{REFERENCES}

KINTSCH, W. (1988). The role of knowledge in discourse processing: A construction-integration model. Psychological Review, 95, 163-182. doi:10.1037/0033-295X.95.2.163

MAYER, R. E. (1983). Can you repeat that? Qualitative effects of repetition and advance organizers on learning from science prose. Journal of Educational Psychology, 75, 40-49. doi:10.1037/0022-0663.75.1.40

MAYER, R. E. (2009). Multimedia learning (2nd ed.). New York: Cambridge University Press.

Millis, K. K., Simon, S., \& TenBroek, N. S. (1998). Resource allocation during the rereading of scientific texts. Memory \& Cognition, 26, 232-246.

PACKer, R., \& Jordan, K. (2001). Overture. In R. Packer \& K. Jordan (Eds.), Multimedia: From Wagner to virtual reality (pp. xv-xxxviii). New York: Norton.

Rawson, K. A., \& Kintsch, W. (2004). Exploring encoding and retrieval effects of background information on text memory. Discourse Processes, 38, 323-344. doi:10.1207/s15326950dp3803_3

WAdDILl, P. J., \& MCDANiEL, M. A. (1992). Pictorial enhancement of text memory: Limitations imposed by picture type and comprehension skill. Memory \& Cognition, 20, 472-482.

(Manuscript received March 7, 2009; revision accepted for publication September 17, 2009.) 